\title{
Academic performance and social factors related to cigarette smoking by schoolchildren
}

\author{
BEULAH R. BEWLEY AND J. M. BLAND* \\ From the Department of Community Medicine, St Thomas's Hospital Medical School
}

SUMMARY Factors which may influence boys and girls aged between 10 and $12 \frac{1}{2}$ years to star屌 smoking were studied. Information was obtained from 491 schoolchildren, their parents, and headteachers. In their own view and that of their headteachers children who did not smoke were academically better than smokers. Children who smoked were more likely than non-smokers tơ have a parent and siblings of the same sex who smoked. No association was found between the child's own smoking and that of parents and siblings of the opposite sex. Smokers were more likely to have friends who smoked. Most children did not think smoking was enjoyable or desirable ando many thought it bad for health, irrespective of their own smoking habits. The majority thought people of their own age smoked to show off.

Despite years of research into cigarette smoking and propaganda against it, many adults continue to smoke, and more young people take up the habit every year (Royal College of Physicians, 1971; Todd, 1972).

Several British studies have examined the background to smoking in children (Public Health Department Study Group, 1959; Cartwright and Thomson, 1960; McKennell and Thomas, 1967; Bynner, 1969) and it would seem that it is associated with a number of factors. These include smoking by parents, siblings, and friends, anticipation of adulthood, low academic achievement, attending secondary modern school, having parents from social classes IV and $\mathrm{V}$, and living in an urban environment. However, they may be discouraged from smoking by health education, parental example, and sanctions.

To find out more about why young children take up smoking two studies have been carried out. In the first, Bewley et al. (1974) studied 229 Derbyshire boys in their final year at primary school. The study sought to find any relationship between the boys' smoking and that of their parents, siblings, and friends, the boys' own attitudes to smoking, and the reasons why they thought children smoked. In the current study smoking habits of boys and girls are compared, and academic performance related to smoking, parents' attitude to smoking, and the repeatability of the previous findings are investigated.

* Present address: Department of Clinical Epidemiology and Social Medicine, St George's Hospital Medical School, London

\section{Method}

In November 1971, 4804 children aged between $10 . \mathrm{N}$ and $12 \frac{1}{2}$ years, in the final year of primary schogl or the first year of secondary school in Ke completed a short self-administered questionnaire on their smoking habits and respiratory symptoms (Bewley and Bland, 1976). The children were classified into four groups: heavy smokers, smoking one or more cigarettes a day; light smokers, smoking을 one or more a week; experimental smokers, who had $\overrightarrow{ }$ smoked but were currently smoking less than one cigarette a week; and non-smokers, who had never smoked. A sample of 501 children, stratified by amount smoked, was selected for further study. All the heavy smokers found in the first stage 3 were included, and a further group of light smokersi chosen at random. These 167 smokers were then matched for sex, school class, and age (within six


non-smokers, chosen at random. In June 1972, $\frac{D}{O}$ these children were asked to complete a second, more detailed, questionnaire at school under the $\tilde{N}$ direction of five trained health visitors. Each child was asked about his own smoking habits and those $\mathrm{N}$ of family and friends, details about his first $\omega$ cigarette, beliefs about why children do or do not smoke, and his attitude to smoking and health. At $\stackrel{\circ}{\circ}$ the same time, the headteacher in the school was $\mathbb{D}$ asked to rate each child's school work as good,? average, or poor compared with other children in ${ }^{\circ}$ the class. Neither the headteacher nor the health visitor was told which children smoked. 
In August 1972, the parents of these children were sent a self-administered questionnaire requesting information on the child's past respiratory illnesses and symptoms, parents' occupation, smoking habits, and attitude to children's smoking. Parents who failed to reply after two follow-up postal questionnaires were visited by one of the health visitors.

\section{Results}

\section{RESPONSE RATES AND CONSISTENCY}

Questionnaires were completed by 491 children (98\% response rate) and 450 parents $(92 \%$ response rate).

In both questionnaires the children were asked about their smoking, and $63 \%$ remained in the same category. As some change in smoking could be expected over the seven-month period between the first and second questionnaire the only reliable check of consistency was to consider those who, in November 1971, reported having smoked at least once. In June $1972,92 \%$ of these again reported having smoked. The result will be reported here in terms of original classification.

Both children and parents were asked whether parents smoked. Of the parents who returned questionnaires, $90 \%$ confirmed the child's reply about their own smoking. The remaining $10 \%$ included cases where there was no father or mother. There were discrepancies in 49 cases where children reported that a parent smoked while the parent reported being a non-smoker, and in 13 cases where children reported a parent to be a non-smoker when the parents reported himself or herself as a smoker.

\section{SMOKING BY PARENTS}

Table 1 shows parents' smoking as reported by their children. The data from the parents showed the same pattern. Children were more likely to smoke if their parents smoked. Boys' smoking was related to their fathers' but not to their mothers' smoking. For girls, the association was with mothers' but not with fathers' smoking.

\section{BROTHERS' AND SISTERS' SMOKING}

Table 2 shows smoking by brothers as reported by the children. For boys, there was no relationship between having brothers and smoking, but there was a strong relationship between having brothers who smoked and the boys' own smoking. Forty-five per cent of boys who were heavy smokers had brothers who smoked, compared with $7 \%$ of nonsmokers. There was little evidence for girls that the number of brothers or their smoking was associated with the girls' smoking.

The relationship with brothers' smoking could be a reflection of the influence of the fathers' smoking. Table 3 shows the smoking of both father and brothers related to the boys' own smoking. More smokers than non-smoliers had brothers who smoked, both among boys whose fathers were non-smokers and boys whose fathers smoked. However, only among boys whose fathers smoked were there many smoking brothers. Although the brother appears to be an important influence, it is only likely to be so if the father smokes.

The smoking habits of sisters is shown in Table 4. For the boys, smoking by sisters was significantly associated with their own smoking; but $54 \%$ of boys with a sister who smoked also had a brother who smoked, compared with $16 \%$ of boys with no

Table 1 Smoking by parents (as reported by the children)

\begin{tabular}{|c|c|c|c|c|c|c|c|c|c|c|c|c|}
\hline \multirow{3}{*}{ Questionnaire } & \multirow{3}{*}{$\begin{array}{l}\text { Parents' } \\
\text { smoking }\end{array}$} & \multicolumn{4}{|c|}{ Smokers } & \multirow{2}{*}{\multicolumn{2}{|c|}{$\begin{array}{l}\text { Experimental } \\
\text { smokers }\end{array}$}} & \multirow{2}{*}{\multicolumn{2}{|c|}{ Non-smokers }} & \multirow{2}{*}{\multicolumn{2}{|c|}{ Total }} & \multirow{3}{*}{ Test of significance* } \\
\hline & & \multicolumn{2}{|c|}{ Heavy } & \multicolumn{2}{|c|}{ Light } & & & & & & & \\
\hline & & No. & $\%$ & No. & $\%$ & No. & $\%$ & No. & $\%$ & No. & $\%$ & \\
\hline \multirow[t]{3}{*}{ Boys } & $\begin{array}{l}\text { Father } \\
\text { smokes } \\
\text { non-smoker }\end{array}$ & $\begin{array}{r}25 \\
6\end{array}$ & $\begin{array}{l}80 \cdot 6 \\
19 \cdot 4\end{array}$ & $\begin{array}{l}65 \\
21\end{array}$ & $\begin{array}{l}75 \cdot 6 \\
24 \cdot 4\end{array}$ & $\begin{array}{l}89 \\
34\end{array}$ & $\begin{array}{l}72 \cdot 4 \\
27 \cdot 6\end{array}$ & $\begin{array}{l}71 \\
52\end{array}$ & $\begin{array}{l}57 \cdot 7 \\
42 \cdot 3\end{array}$ & $\begin{array}{l}250 \\
113\end{array}$ & $\begin{array}{l}68 \cdot 9 \\
31 \cdot 1\end{array}$ & $\begin{array}{l}\chi^{2}=11.64, \text { df }=3 \\
P>0.05\end{array}$ \\
\hline & $\begin{array}{l}\text { Mother } \\
\text { smokes } \\
\text { non-smoker }\end{array}$ & $\begin{array}{l}17 \\
14\end{array}$ & $\begin{array}{l}54 \cdot 8 \\
45 \cdot 2\end{array}$ & $\begin{array}{l}45 \\
41\end{array}$ & $\begin{array}{l}52 \cdot 3 \\
47 \cdot 7\end{array}$ & $\begin{array}{l}62 \\
61\end{array}$ & $\begin{array}{l}50 \cdot 4 \\
49 \cdot 6\end{array}$ & $\begin{array}{l}60 \\
63\end{array}$ & $\begin{array}{l}48 \cdot 8 \\
51 \cdot 2\end{array}$ & $\begin{array}{l}184 \\
179\end{array}$ & $\begin{array}{l}50 \cdot 7 \\
49 \cdot 3\end{array}$ & $\begin{array}{l}\chi^{8}=0.49, \text { df }=3 \\
P>0.50\end{array}$ \\
\hline & & 31 & $100 \cdot 0$ & 86 & $100 \cdot 0$ & 123 & $100 \cdot 0$ & 123 & $100 \cdot 0$ & 363 & $100 \cdot 0$ & \\
\hline \multirow[t]{3}{*}{ Girls } & $\begin{array}{l}\text { Father } \\
\text { smokes } \\
\text { non-smoker } \\
\text { Not known } \\
\text { Mother }\end{array}$ & $\begin{array}{l}1 \\
1 \\
0\end{array}$ & $\begin{array}{l}50.0 \\
50.0 \\
00.0\end{array}$ & $\begin{array}{r}23 \\
17 \\
0\end{array}$ & $\begin{array}{l}57 \cdot 5 \\
42 \cdot 5 \\
00 \cdot 0\end{array}$ & $\begin{array}{r}22 \\
20 \\
1\end{array}$ & $\begin{array}{r}51 \cdot 2 \\
46 \cdot 5 \\
2 \cdot 3\end{array}$ & $\begin{array}{r}23 \\
20 \\
0\end{array}$ & $\begin{array}{l}53 \cdot 5 \\
46 \cdot 5 \\
00 \cdot 0\end{array}$ & $\begin{array}{r}69 \\
58 \\
1\end{array}$ & $\begin{array}{r}53 \cdot 9 \\
45 \cdot 3 \\
0 \cdot 8\end{array}$ & $\begin{array}{l}x^{2}=0 \cdot 21, \text { df }=2 \\
P>0 \cdot 50\end{array}$ \\
\hline & $\begin{array}{l}\text { smokes } \\
\text { non-smoker } \\
\text { Not known }\end{array}$ & $\begin{array}{l}1 \\
1 \\
0\end{array}$ & $\begin{array}{l}50.0 \\
50.0 \\
00.0\end{array}$ & $\begin{array}{r}29 \\
11 \\
0\end{array}$ & $\begin{array}{l}72 \cdot 5 \\
27 \cdot 5 \\
00 \cdot 0\end{array}$ & $\begin{array}{r}22 \\
20 \\
1\end{array}$ & $\begin{array}{r}51 \cdot 2 \\
46 \cdot 5 \\
2 \cdot 3\end{array}$ & $\begin{array}{r}16 \\
27 \\
0\end{array}$ & $\begin{array}{l}37 \cdot 2 \\
62 \cdot 8 \\
00 \cdot 0\end{array}$ & $\begin{array}{r}68 \\
59 \\
1\end{array}$ & $\begin{array}{r}53 \cdot 1 \\
46 \cdot 1 \\
0 \cdot 8\end{array}$ & $\begin{array}{l}x^{2}=10.04, \text { df }=2 \\
P<0.01\end{array}$ \\
\hline & & 2 & $100 \cdot 0$ & 40 & $100 \cdot 0$ & 43 & $100 \cdot 0$ & 43 & $100 \cdot 0$ & 128 & $100 \cdot 0$ & \\
\hline
\end{tabular}

*Test for association between parents' and child's smoking. For the girls, heavy and light smokers were combined to give a $2 \times 3$ table. 
Table 2 Smoking habit of brothers

\begin{tabular}{|c|c|c|c|c|c|c|c|c|c|c|c|c|}
\hline & \multirow{3}{*}{$\begin{array}{l}\text { No. of brothers } \\
\text { who smoke }\end{array}$} & \multicolumn{4}{|c|}{ Smokers } & \multirow{2}{*}{\multicolumn{2}{|c|}{$\begin{array}{l}\text { Experimental } \\
\text { smokers }\end{array}$}} & \multirow{2}{*}{\multicolumn{2}{|c|}{ Non-smokers }} & \multirow{2}{*}{\multicolumn{2}{|c|}{ Total }} & \multirow{3}{*}{ Significance of association* } \\
\hline & & \multicolumn{2}{|c|}{ Heavy } & \multicolumn{2}{|c|}{ Light } & & & & & & & \\
\hline & & No. & $\%$ & No. & $\%$ & No. & $\%$ & No. & $\%$ & No. & $\%$ & \\
\hline \multirow[t]{2}{*}{ Boys } & $\begin{array}{l}\text { None } \\
\text { One or more, } \\
\text { but non-smokers }\end{array}$ & 12 & $16 \cdot 1$ & 19 & $22 \cdot 1$ & 43 & $35 \cdot 0$ & 43 & $35 \cdot 0$ & 110 & $48 \cdot 5$ & \multirow{2}{*}{$\begin{array}{l}\text { Boys with no brothers compared } \\
\text { to boys with brothers but } \\
\text { all non-smokers: } \chi^{2}=2 \cdot 31 \text {, } \\
\text { df }=2,0 \cdot 25<P<0 \cdot 50 \\
\text { Boys with either no brothers, or } \\
\text { with brothers none of whom } \\
\text { smoke, compared to boys with } \\
\text { at least } 1 \text { brother who smokes } \\
\chi^{2}=29 \cdot 34 \text {, df }=2, P<0.001\end{array}$} \\
\hline & $\begin{array}{l}\text { One or more, at } \\
\text { least one smoker } \\
\text { Not known }\end{array}$ & $\begin{array}{r}14 \\
0 \\
31\end{array}$ & $\begin{array}{r}45 \cdot 2 \\
00 \cdot 0 \\
100 \cdot 0\end{array}$ & $\begin{array}{r}28 \\
0 \\
86\end{array}$ & $\begin{array}{r}32 \cdot 6 \\
00 \cdot 0 \\
100 \cdot 0\end{array}$ & $\begin{array}{r}25 \\
0 \\
123\end{array}$ & $\begin{array}{r}20 \cdot 3 \\
00 \cdot 0 \\
100 \cdot 0\end{array}$ & $\begin{array}{r}9 \\
1 \\
123\end{array}$ & $\begin{array}{r}7 \cdot 3 \\
0 \cdot 8 \\
100 \cdot 0\end{array}$ & $\begin{array}{r}76 \\
1 \\
363\end{array}$ & $\begin{array}{r}20 \cdot 9 \\
0 \cdot 3 \\
100 \cdot 0\end{array}$ & \\
\hline \multirow[t]{2}{*}{ Girls } & $\begin{array}{l}\text { None } \\
\text { One or more, } \\
\text { but non-smokers }\end{array}$ & 1 & $50 \cdot 0$ & 16 & $40 \cdot 0$ & 13 & $58 \cdot 2$ & 12 & $60 \cdot 5$ & 68 & $30 \cdot 5$ & $\begin{array}{l}\text { Girls with no brothers compared } \\
\text { to girls with brothers but all } \\
\text { non-smokers: } \chi^{2}=1 \cdot 39 \text {, } \\
\text { df }=2,0.25<P<0.50\end{array}$ \\
\hline & $\begin{array}{l}\text { One or more, at } \\
\text { least one smoker } \\
\text { Not known }\end{array}$ & $\begin{array}{l}0 \\
0 \\
2\end{array}$ & $\begin{array}{r}00 \cdot 0 \\
00 \cdot 0 \\
100 \cdot 0\end{array}$ & $\begin{array}{r}11 \\
0 \\
40\end{array}$ & $\begin{array}{r}27 \cdot 5 \\
00 \cdot 0 \\
100 \cdot 0\end{array}$ & $\begin{array}{r}4 \\
1 \\
43\end{array}$ & $\begin{array}{r}9 \cdot 3 \\
2 \cdot 3 \\
100 \cdot 0\end{array}$ & $\begin{array}{r}5 \\
0 \\
43\end{array}$ & $\begin{array}{r}11 \cdot 6 \\
00 \cdot 0 \\
100 \cdot 0\end{array}$ & $\begin{array}{r}20 \\
1 \\
128\end{array}$ & $\begin{array}{r}15 \cdot 6 \\
0 \cdot 8 \\
100 \cdot 0\end{array}$ & $\begin{array}{l}\text { Girls with either no brothers, or } \\
\text { with brothers none of whom } \\
\text { smoke, compared to girls with a } \\
\text { least } 1 \text { brother who smokes: } \\
\chi^{2}=5 \cdot 23 \text {, df }=2 \text {, } \\
0.05<P<0.10\end{array}$ \\
\hline
\end{tabular}

Table 3 Relationship between boys' smoking habits and those of their fathers and brothers

\begin{tabular}{lcccc} 
& \multicolumn{2}{c}{ Boys' smoking habits } & \\
\cline { 2 - 4 } & Smoker & $\begin{array}{l}\text { Experi- } \\
\text { mental } \\
\text { smoker }\end{array}$ & $\begin{array}{l}\text { Non- } \\
\text { smoker }\end{array}$ & Total \\
\hline $\begin{array}{l}\text { Father does not smoke } \\
\begin{array}{l}\text { No brothers who smoke } \\
\text { At least one brother who }\end{array}\end{array}$ & 19 & 31 & 50 & 100 \\
$\begin{array}{l}\text { smokes } \\
\text { Father smokes }\end{array}$ & 8 & 3 & 2 & 13 \\
$\begin{array}{l}\text { No brothers who smoke } \\
\text { At least one brother who }\end{array}$ & 56 & 67 & 63 & 186 \\
$\begin{array}{l}\text { smokes } \\
\text { Not known }\end{array}$ & 34 & 22 & 7 & 63 \\
Total & 0 & 0 & 1 & 1 \\
\hline
\end{tabular}

sisters who smoked. Table 5 shows that for boys who had no brothers who smoked, there was no relationship between the boys' own smoking and that of their sisters. Conversely, when the boys were grouped by their sisters' smoking, there was a significant relationship between their own smoking and their brothers'. For the girls there was a significant association between their own and their sisters' smoking. There were insufficient girls in the study to enable the separate effect of mothers' and sisters' smoking to be investigated.

\section{SMOKING BY FRIENDS}

The children were asked how many of their friends smoked. Seventy-six per cent of children who were smokers reported that most or some of their school friends smoked, compared with $36 \%$ of non-smokers.
The smokers reported that more of their friengs smoked and were more certain about their friencs. smoking than the non-smokers. More boys thenio girls said their friends smoked, and fewer giflss knew how many of their friends smoked. Smoking reported for friends outside school showed a similarô pattern.

\section{THE FIRST CIGARETTE}

Table 6 shows the age at which children tried their first cigarette. Sixteen per cent of the boys claimed? this was at six or younger, so these figures should be treated with some caution. The boys were? younger when they started smoking than the girls.

Home was the most common place for the first. cigarette, especially for girls, and it was usuallyo obtained from friends or family. More often this cigarette was smoked with friends than with parents? or siblings or when alone, but $22 \%$ of children $>$ who had smoked said that their first cigarette was smoked with their parents.

REASONS FOR AND AGAINST SMOKING
The children were asked why they thought people of their own age did or did not smoke. They were asked to choose as many reasons as they wished fromo a list. There were only slight differences between ${ }_{\bar{\Phi}}^{-}$ answers from smokers and non-smokers, or from? boys and girls.

The most frequent reason for smoking was 'theyo want to show off or look big', chosen by $62 \%$ of the children. 'To be grown up' was chosen by $8 \%$ of 
Table 4 No. of sisters who smoke

\begin{tabular}{|c|c|c|c|c|c|c|c|c|c|c|c|c|}
\hline & \multirow{3}{*}{$\begin{array}{l}\text { No. of sisters } \\
\text { who smoke }\end{array}$} & \multicolumn{4}{|c|}{ Smokers } & \multirow{2}{*}{\multicolumn{2}{|c|}{$\begin{array}{l}\text { Experimental } \\
\text { smokers }\end{array}$}} & \multirow{2}{*}{\multicolumn{2}{|c|}{ Non-smokers }} & \multirow{2}{*}{\multicolumn{2}{|c|}{ Total }} & \multirow{3}{*}{ Significance of association* } \\
\hline & & \multicolumn{2}{|c|}{ Heavy } & \multicolumn{2}{|c|}{ Light } & & & & & & & \\
\hline & & No. & $\%$ & No. & $\%$ & No. & $\%$ & No. & $\%$ & No. & $\%$ & \\
\hline \multirow[t]{5}{*}{ Boys } & None & 8 & $25 \cdot 8$ & 30 & $34 \cdot 9$ & 36 & $29 \cdot 3$ & 46 & $37 \cdot 4$ & 120 & $33 \cdot 0$ & \multirow{2}{*}{$\begin{array}{l}\text { Boys with no sisters compared to } \\
\text { boys with sisters but all non- } \\
\text { smokers: } x^{2}=1.51 \text {, df }=2 \text {. } \\
0.25<\text { P }<0.50\end{array}$} \\
\hline & $\begin{array}{l}\text { One or more, } \\
\text { but non-smokers }\end{array}$ & 14 & $45 \cdot 2$ & 41 & $47 \cdot 7$ & 72 & $58 \cdot 5$ & 69 & $56 \cdot 1$ & 196 & $54 \cdot 0$ & \\
\hline & $\begin{array}{l}\text { One or more, at } \\
\text { least one smoker }\end{array}$ & 9 & $29 \cdot 0$ & 15 & $17 \cdot 4$ & 15 & $12 \cdot 2$ & 7 & $5 \cdot 7$ & 46 & $12 \cdot 7$ & \multirow{3}{*}{$\begin{array}{l}\text { Boys with either no sisters or } \\
\text { with sisters none of whom smoke } \\
\text { compared to boys with } \\
\text { sisters at least } 1 \text { of whom smokes: } \\
\chi^{2}=11 \cdot 8, \text { df }=2, P<0.01\end{array}$} \\
\hline & \multirow[t]{2}{*}{ Not known } & 0 & $00 \cdot 0$ & $\mathbf{0}$ & $00 \cdot 0$ & 0 & $00 \cdot 0$ & 1 & 0.8 & 1 & $0 \cdot 3$ & \\
\hline & & 31 & $100 \cdot 0$ & 86 & $100 \cdot 0$ & 123 & $100 \cdot 0$ & 123 & $100 \cdot 0$ & 363 & $100 \cdot 0$ & \\
\hline \multirow[t]{5}{*}{ Girls } & None & 0 & $00 \cdot 0$ & 12 & $30 \cdot 0$ & 18 & $41 \cdot 9$ & 22 & $51 \cdot 1$ & 52 & $40 \cdot 6$ & \multirow{2}{*}{$\begin{array}{l}\text { Girls with no sisters compared to } \\
\text { girls with sisters but all non- } \\
\text { smokers: } \chi^{2}=1.85 \text {, df }=2 \text {, } \\
0 \cdot 25<\mathbf{P}<0 \cdot 50\end{array}$} \\
\hline & $\begin{array}{l}\text { One or more, } \\
\text { but non-smokers }\end{array}$ & 2 & $100 \cdot 0$ & 18 & $45 \cdot 0$ & 19 & $44 \cdot 2$ & 19 & $44 \cdot 2$ & 58 & $45 \cdot 3$ & \\
\hline & $\begin{array}{l}\text { One or more, at } \\
\text { least one smoker }\end{array}$ & $\mathbf{0}$ & $00 \cdot 0$ & 10 & $25 \cdot 0$ & 5 & $11 \cdot 6$ & 2 & $4 \cdot 7$ & 17 & $13 \cdot 3$ & \multirow{3}{*}{$\begin{array}{l}\text { Girls with either no sisters or } \\
\text { with sisters none of whom smoke } \\
\text { compared to girls with sisters at } \\
\text { least } 1 \text { of whom smokes: } \\
\chi^{2}=6.85, \text { df }=2, P<0.05\end{array}$} \\
\hline & \multirow[t]{2}{*}{ Not known } & 0 & $00 \cdot 0$ & 0 & $00 \cdot 0$ & 1 & $2 \cdot 3$ & 0 & $00 \cdot 0$ & 1 & 0.8 & \\
\hline & & 2 & $100 \cdot 0$ & 40 & $100 \cdot 0$ & 43 & $100 \cdot 0$ & 43 & $100 \cdot 0$ & 128 & $100 \cdot 0$ & \\
\hline
\end{tabular}

${ }^{*} \chi^{2}$ for the whole table has been partitioned using the formulae of Kimball (1954). Heavy and light smokers have been combined to give a $3 \times 3$ table.

Table 5 Relationship between boys' smoking habits and those of their brothers and sisters

\begin{tabular}{|c|c|c|c|c|}
\hline & \multicolumn{3}{|c|}{ Boys' smoking habits } & \multirow[b]{2}{*}{ Tota } \\
\hline & Smoker & $\begin{array}{l}\text { Experi- } \\
\text { mental } \\
\text { smoker }\end{array}$ & $\begin{array}{l}\text { Non- } \\
\text { smoker }\end{array}$ & \\
\hline \multirow{2}{*}{$\begin{array}{l}\text { No brothers who smoke } \\
\text { No sisters who smoke } \\
\text { At least one sister who } \\
\text { smokes }\end{array}$} & 68 & 89 & 108 & 265 \\
\hline & 7 & 9 & 5 & 21 \\
\hline \multirow{3}{*}{$\begin{array}{l}\text { At least one brother who } \\
\text { smokes } \\
\text { No sisters who smoke } \\
\text { At least one sister who } \\
\text { smokes }\end{array}$} & & & & \\
\hline & 25 & 19 & 7 & 51 \\
\hline & 17 & 6 & 2 & 25 \\
\hline Not known & 0 & o & 1 & 1 \\
\hline
\end{tabular}

the children. Fewer gave the example of others as a reason, $35 \%$ choosing 'Because their friends smoke' and $27 \%$ 'Because their parents smoke'. The children did not think the actual act of smoking was important, 'Because they like it' was chosen by $24 \%$ and 'They think it is relaxing' by $9 \%$. Only $7 \%$ thought children smoked 'Because they are curious'.

The most common reason for not smoking was 'They are worried because smoking is bad for their health', which was chosen by $71 \%$ of the children. Pressure from adults was chosen next, 'Parents tell them not to' being chosen by $46 \%$ and 'They would get into trouble if they did' by $34 \%$. Fewer children thought teachers were important, 'Teachers tell them not to' being chosen by $24 \%$. 'It is expensive' was chosen by $36 \%$, 'It is a dirty habit' by $30 \%$, and 'It is a waste of time' by $23 \%$. The
Table 6 Factors associated with first cigarette, for all children who had tried smoking by stage 2

\begin{tabular}{|c|c|c|c|c|}
\hline \multirow{2}{*}{ First cigarette } & \multicolumn{2}{|c|}{ Boys } & \multicolumn{2}{|l|}{ Girls } \\
\hline & No. & $\%$ & No. & $\%$ \\
\hline $\begin{array}{l}\text { Age (years) } \\
\text { At six or less } \\
\text { seven } \\
\text { eight } \\
\text { nine } \\
\text { ten } \\
\text { eleven or more } \\
\text { Not known }\end{array}$ & $\begin{array}{r}43 \\
28 \\
39 \\
60 \\
59 \\
33 \\
7\end{array}$ & $\begin{array}{l}16 \cdot 0 \\
10 \cdot 4 \\
14 \cdot 5 \\
22 \cdot 3 \\
21 \cdot 9 \\
12 \cdot 3 \\
2 \cdot 6\end{array}$ & $\begin{array}{r}6 \\
5 \\
5 \\
18 \\
30 \\
19 \\
0\end{array}$ & $\begin{array}{r}7 \cdot 2 \\
6 \cdot 0 \\
6 \cdot 0 \\
21 \cdot 7 \\
36 \cdot 2 \\
22 \cdot 9 \\
00 \cdot 0\end{array}$ \\
\hline $\begin{array}{l}\text { Place } \\
\text { At home } \\
\text { Elsewhere } \\
\text { Not known }\end{array}$ & $\begin{array}{r}80 \\
183 \\
6\end{array}$ & $\begin{array}{r}29 \cdot 7 \\
68 \cdot 1 \\
2 \cdot 2\end{array}$ & $\begin{array}{r}40 \\
43 \\
0\end{array}$ & $\begin{array}{l}48 \cdot 2 \\
51 \cdot 8 \\
00 \cdot 0\end{array}$ \\
\hline $\begin{array}{l}\text { Source } \\
\text { From family } \\
\text { friend } \\
\text { other } \\
\text { Not known }\end{array}$ & $\begin{array}{r}73 \\
131 \\
59 \\
6\end{array}$ & $\begin{array}{r}27 \cdot 2 \\
48 \cdot 7 \\
21 \cdot 9 \\
2 \cdot 2\end{array}$ & $\begin{array}{r}31 \\
31 \\
21 \\
0\end{array}$ & $\begin{array}{r}37 \cdot 4 \\
37 \cdot 4 \\
25 \cdot 3 \\
0 \cdot 0\end{array}$ \\
\hline $\begin{array}{l}\text { Company } \\
\text { Alone } \\
\text { With friends } \\
\text { brother or sister } \\
\text { parent } \\
\text { other } \\
\text { Not known }\end{array}$ & $\begin{array}{r}34 \\
130 \\
35 \\
52 \\
12 \\
6\end{array}$ & $\begin{array}{r}12 \cdot 7 \\
48 \cdot 3 \\
13 \cdot 0 \\
19 \cdot 3 \\
4 \cdot 5 \\
2 \cdot 2\end{array}$ & $\begin{array}{r}8 \\
31 \\
14 \\
23 \\
7 \\
0\end{array}$ & $\begin{array}{r}9 \cdot 6 \\
37 \cdot 4 \\
16 \cdot 9 \\
27 \cdot 7 \\
8 \cdot 4 \\
0 \cdot 0\end{array}$ \\
\hline Total & 269 & $100 \cdot 0$ & 83 & $100 \cdot 0$ \\
\hline
\end{tabular}

children did not seem to think peer group pressure was as important, only $14 \%$ choosing 'Other children they know do not smoke'.

\section{ATTITUDES TO SMOKING}

The children were asked whether they agreed or disagreed with a number of statements on attitude. The results are shown in Table 7. As there was little difference in the replies of boys and girls, these have not been presented separately. Overall, $87 \%$ 
Table 7 No. of children agreeing with statements about smoking

\begin{tabular}{|c|c|c|c|c|c|c|c|c|c|c|c|c|}
\hline \multirow{3}{*}{ Statement } & \multicolumn{4}{|c|}{ Smokers } & \multirow{2}{*}{\multicolumn{2}{|c|}{$\begin{array}{l}\text { Experimental } \\
\text { smokers }\end{array}$}} & \multirow{2}{*}{\multicolumn{2}{|c|}{ Non-smokers }} & \multirow{2}{*}{\multicolumn{2}{|c|}{ Total }} & \multirow{3}{*}{\multicolumn{2}{|c|}{$\begin{array}{l}\text { Association between } \\
\text { attitude and smoking* }\end{array}$}} \\
\hline & \multicolumn{2}{|c|}{ Heavy } & \multicolumn{2}{|c|}{ Light } & & & & & & & & \\
\hline & No. & $\%$ & No. & $\%$ & No. & $\%$ & No. & $\%$ & No. & $\%$ & & \\
\hline Smoking is bad for your health & 31 & $93 \cdot 9$ & 107 & $84 \cdot 9$ & 151 & $91 \cdot 0$ & 158 & $95 \cdot 2$ & 447 & $91 \cdot 0$ & $9 \cdot 6$ & $P=0.05$ \\
\hline Smoking causes cancer & 27 & $81 \cdot 8$ & 106 & $84 \cdot 1$ & 151 & $91 \cdot 0$ & 156 & $94 \cdot 0$ & 440 & $89 \cdot 6$ & $9 \cdot 9$ & $\mathrm{P}<0.05$ \\
\hline $\begin{array}{l}\text { Parents should try to stop their } \\
\text { children from smoking }\end{array}$ & 27 & $81 \cdot 8$ & 101 & $80 \cdot 2$ & 151 & $91 \cdot 0$ & 154 & $92 \cdot 8$ & 433 & $88 \cdot 2$ & $13 \cdot 7$ & $P<0.01$ \\
\hline $\begin{array}{l}\text { It is a bad idea for children } \\
\text { to smoke }\end{array}$ & 24 & $72 \cdot 7$ & 100 & $79 \cdot 4$ & 147 & $88 \cdot 6$ & 158 & $95 \cdot 2$ & 429 & $87 \cdot 4$ & $23 \cdot 1$ & $P<0.001$ \\
\hline Smoking is a waste of money & 28 & $84 \cdot 8$ & 86 & $68 \cdot 3$ & 139 & $83 \cdot 7$ & 147 & $88 \cdot 6$ & 400 & $81 \cdot 5$ & $20 \cdot 9$ & $P<0.001$ \\
\hline $\begin{array}{l}\text { People of my age smoke to } \\
\text { show off }\end{array}$ & 23 & $69 \cdot 7$ & 82 & $65 \cdot 1$ & 133 & $80 \cdot 1$ & 150 & $90 \cdot 4$ & 388 & $79 \cdot 0$ & $29 \cdot 5$ & $P<0.001$ \\
\hline $\begin{array}{l}\text { Smoking makes you feel } \\
\text { grown up }\end{array}$ & 10 & $30 \cdot 3$ & 55 & $43 \cdot 7$ & 64 & $38 \cdot 6$ & 64 & $38 \cdot 6$ & 193 & $39 \cdot 3$ & $2 \cdot 2$ & $0.5<P<0.7$ \\
\hline $\begin{array}{l}\text { It is nice to smoke with } \\
\text { your friends }\end{array}$ & 16 & $48 \cdot 5$ & 54 & $42 \cdot 9$ & 48 & $28 \cdot 9$ & 23 & $13 \cdot 9$ & 141 & $28 \cdot 7$ & $36 \cdot 5$ & $P<0.001$ \\
\hline Smoking is enjoyable & 12 & $36 \cdot 4$ & 44 & $34 \cdot 9$ & 28 & $16 \cdot 9$ & 11 & $6 \cdot 6$ & 95 & $19 \cdot 3$ & $43 \cdot 6$ & $P<0.001$ \\
\hline Total & 33 & $100 \cdot 0$ & 126 & $100 \cdot 0$ & 166 & $100 \cdot 0$ & 166 & $100 \cdot 0$ & 491 & $100 \cdot 0$ & & \\
\hline
\end{tabular}

${ }^{*} \chi^{2}$ or 3 degrees of freedom.

Table 8 Child's assessment of own schoolwork

\begin{tabular}{|c|c|c|c|c|c|c|c|c|c|c|c|c|c|}
\hline & \multirow{3}{*}{ Assessment } & \multicolumn{4}{|c|}{ Smokers } & \multirow{2}{*}{\multicolumn{2}{|c|}{$\begin{array}{l}\text { Experimental } \\
\text { smokers }\end{array}$}} & \multirow{2}{*}{\multicolumn{2}{|c|}{ Non-smokers }} & \multirow{2}{*}{\multicolumn{2}{|c|}{ Total }} & \multirow{3}{*}{ Significance* } & \multirow{3}{*}{ 음 } \\
\hline & & \multicolumn{2}{|c|}{ Heavy } & \multicolumn{2}{|c|}{ Light } & & & & & & & & \\
\hline & & No. & $\%$ & No. & $\%$ & No. & $\%$ & No. & $\%$ & No. & $\%$ & & \\
\hline \multirow[t]{2}{*}{ Boys } & $\begin{array}{l}\text { Good } \\
\text { Average } \\
\text { Poor }\end{array}$ & $\begin{array}{r}7 \\
21 \\
3\end{array}$ & $\begin{array}{r}22 \cdot 6 \\
67 \cdot 7 \\
9 \cdot 7\end{array}$ & $\begin{array}{r}17 \\
64 \\
5\end{array}$ & $\begin{array}{r}19 \cdot 8 \\
74 \cdot 4 \\
5 \cdot 8\end{array}$ & $\begin{array}{r}46 \\
74 \\
3\end{array}$ & $\begin{array}{r}37 \cdot 4 \\
60 \cdot 2 \\
2 \cdot 4\end{array}$ & $\begin{array}{r}41 \\
74 \\
8\end{array}$ & $\begin{array}{r}33 \cdot 3 \\
60 \cdot 2 \\
6 \cdot 5\end{array}$ & $\begin{array}{r}111 \\
233 \\
19\end{array}$ & $\begin{array}{r}30 \cdot 6 \\
64 \cdot 2 \\
5 \cdot 2\end{array}$ & $\begin{array}{l}\chi^{2}=8 \cdot 8, \mathrm{df}=3 \\
\mathrm{P}<0 \cdot 05\end{array}$ & 을 \\
\hline & & 31 & $100 \cdot 0$ & 86 & $100 \cdot 0$ & 123 & $100 \cdot 0$ & 123 & $100 \cdot 0$ & 363 & $100 \cdot 0$ & & \\
\hline \multirow[t]{2}{*}{ Girls } & $\begin{array}{l}\text { Good } \\
\text { Average } \\
\text { Poor } \\
\text { Not known }\end{array}$ & $\frac{-\overline{2}}{-}$ & $\begin{array}{l}100 \cdot 0 \\
-\end{array}$ & $\begin{array}{r}6 \\
27 \\
6 \\
1\end{array}$ & $\begin{array}{r}15 \cdot 0 \\
67 \cdot 5 \\
15 \cdot 0 \\
2 \cdot 5\end{array}$ & $\begin{array}{r}10 \\
32 \\
1 \\
\end{array}$ & $\begin{array}{r}23 \cdot 3 \\
74 \cdot 4 \\
2 \cdot 3 \\
-\end{array}$ & $\begin{array}{r}10 \\
32 \\
1 \\
-\end{array}$ & $\begin{array}{r}23 \cdot 3 \\
74 \cdot 4 \\
2 \cdot 3 \\
-\end{array}$ & $\begin{array}{r}26 \\
93 \\
8 \\
1\end{array}$ & $\begin{array}{r}20 \cdot 3 \\
72 \cdot 6 \\
6 \cdot 3 \\
0 \cdot 8\end{array}$ & $\begin{array}{l}\chi^{2}=1 \cdot 27, \text { df }=2 \\
P<0 \cdot 50\end{array}$ & \\
\hline & & 2 & $100 \cdot 0$ & 40 & $100 \cdot 0$ & 43 & $100 \cdot 0$ & 43 & $100 \cdot 0$ & 128 & $100 \cdot 0$ & & \\
\hline
\end{tabular}

*Test for association between child's assessment and smoking. The poor and average categories have been combined, and for the girls so have heavy and light smokers.

of the children thought it was a bad idea for children to smoke. Significantly more non-smokers than smokers agreed with this, but $73 \%$ of heavy smokers also agreed. Most children agreed that smoking was a waste of money, and that parents should discourage their children from smoking. Nearly all the children thought that smoking was bad for health and that it caused cancer.

Significantly more non-smokers agreed that 'People of my age smoke to show off'. When asked to agree or disagree with the statement 'Smoking makes you feel grown up', there was no difference between the smoking groups.

More smokers than non-smokers thought that it was nice to smoke with friends, and half the heavy smokers agreed with this. Overall, $20 \%$ thought smoking was enjoyable; this was agreed by more smokers than non-smokers although fewer than half the children in any group said this.
ASSESSMENT OF SCHOOL WORK

The children were asked if they thought their school work was good, average, or poor compared with other members of their class. Table 8 shows that most of the children saw their work as good or average; significantly more boys than girls saw their work as good. More non-smokers and experimental smokers said their work was good compared with the smokers; this difference was significant for boys.

The headteachers were asked to classify the children's work using the same criteria. They placed about one-third of the children in each category. As few children rated themselves as poor, these two classifications were not the same. However, they were related, in that children whom the head saw as good tended to see themselves as good, and children who sav'. themselves as poor were also seen as poor by their headteachers. 
Table 9 Headteacher's assessment of schoolwork

\begin{tabular}{|c|c|c|c|c|c|c|c|c|c|c|c|c|}
\hline & \multirow{3}{*}{ Assessment } & \multicolumn{4}{|c|}{ Smokers } & \multirow{2}{*}{\multicolumn{2}{|c|}{$\begin{array}{l}\text { Experimental } \\
\text { smokers }\end{array}$}} & \multirow{2}{*}{\multicolumn{2}{|c|}{ Non-smokers }} & \multirow{2}{*}{\multicolumn{2}{|c|}{ Total }} & \multirow{3}{*}{ Significance* } \\
\hline & & \multicolumn{2}{|c|}{ Heavy } & \multicolumn{2}{|c|}{ Light } & & & & & & & \\
\hline & & No. & $\%$ & No. & $\%$ & No. & $\%$ & No. & $\%$ & No. & $\%$ & \\
\hline \multirow[t]{2}{*}{ Boys } & $\begin{array}{l}\text { Good } \\
\text { Average } \\
\text { Poor } \\
\text { Not known }\end{array}$ & $\begin{array}{r}1 \\
13 \\
15 \\
2\end{array}$ & $\begin{array}{r}3 \cdot 2 \\
41 \cdot 9 \\
48 \cdot 4 \\
6 \cdot 5\end{array}$ & $\begin{array}{l}17 \\
27 \\
32 \\
10\end{array}$ & $\begin{array}{l}19 \cdot 8 \\
31 \cdot 4 \\
37 \cdot 2 \\
11 \cdot 6\end{array}$ & $\begin{array}{l}31 \\
44 \\
35 \\
13\end{array}$ & $\begin{array}{l}25 \cdot 2 \\
35 \cdot 8 \\
28 \cdot 5 \\
10 \cdot 6\end{array}$ & $\begin{array}{l}30 \\
43 \\
35 \\
15\end{array}$ & $\begin{array}{l}24 \cdot 4 \\
35 \cdot 0 \\
28 \cdot 5 \\
12 \cdot 2\end{array}$ & $\begin{array}{r}79 \\
127 \\
117 \\
40\end{array}$ & $\begin{array}{l}27 \cdot 8 \\
35 \cdot 0 \\
32 \cdot 2 \\
11 \cdot 0\end{array}$ & $\begin{array}{l}x^{2}=10.67, \text { df }=6 \\
0.05<P<0.01\end{array}$ \\
\hline & & 31 & $100 \cdot 0$ & 86 & $100 \cdot 0$ & 123 & $100 \cdot 0$ & 123 & $100 \cdot 0$ & 363 & $100 \cdot 0$ & \\
\hline \multirow[t]{2}{*}{ Girls } & $\begin{array}{l}\text { Good } \\
\text { Average } \\
\text { Poor } \\
\text { Not known }\end{array}$ & $\frac{\overline{2}}{-}$ & $\begin{array}{l}\overline{100} \cdot 0 \\
-\end{array}$ & $\begin{array}{r}3 \\
18 \\
16 \\
3\end{array}$ & $\begin{array}{r}7 \cdot 5 \\
45 \cdot 0 \\
40 \cdot 0 \\
7 \cdot 5\end{array}$ & $\begin{array}{r}11 \\
21 \\
9 \\
2\end{array}$ & $\begin{array}{r}25 \cdot 6 \\
48 \cdot 8 \\
20.9 \\
4 \cdot 7\end{array}$ & $\begin{array}{r}15 \\
19 \\
7 \\
2\end{array}$ & $\begin{array}{r}34 \cdot 9 \\
44 \cdot 2 \\
16 \cdot 3 \\
4 \cdot 7\end{array}$ & $\begin{array}{r}29 \\
60 \\
32 \\
7\end{array}$ & $\begin{array}{r}22 \cdot 7 \\
46 \cdot 9 \\
25 \cdot 0 \\
5 \cdot 5\end{array}$ & $\begin{array}{l}\chi^{2}=12.12, \mathrm{df}=4 \\
P<0.05\end{array}$ \\
\hline & & 2 & $100 \cdot 0$ & 40 & $100 \cdot 0$ & 43 & $100 \cdot 0$ & 43 & $100 \cdot 0$ & 128 & $100 \cdot 0$ & \\
\hline
\end{tabular}

*Test for association between headteacher's assessment and child's smoking. Not knowns have been omitted and for girls heavy and light smokers have been combined.

The relationship between the headteacher's assessment and the child's smoking is shown in Table 9. Again, more non-smokers than smokers were good and more smokers than non-smokers were poor.

\section{Discussion}

We have examined some of the characteristics of children aged between 10 and $12 \frac{1}{2}$, some of whom were already smoking. The findings are important because of the age of the subjects.

The young smoker was more likely than the non-smoker to come from a home where there were smokers of the same sex as the child. Smoking by parents and brothers and sisters of the opposite sex did not seem to be strongly related to the child's smoking. The influence of parents' smoking has been well documented (Public Health Department Study Group, 1959; Cartwright and Thomson, 1960; Salber et al., 1968; Palmer, 1970). An association between boys' smoking and fathers' smoking was also found by O'Rourke and WilsonDavies (1970) and Bewley et al. (1974), but not by Bynner (1969). No association between boys' smoking and their mothers' smoking was found in Derbyshire (Bewley et al., 1974), although other workers have reported that the mothers' smoking was important (Bothwell, 1959; Lemin, 1966). The importance of the father and lack of influence of the mother found for boys in this study was not true for girls, the opposite being the case. It would seem that the smoking habits of the parent of the same sex as the child is important in this age group.

The association between boys' smoking and their brothers' has been reported (Lemin, 1966; Bynner, 1969; Bewley et al., 1974). This study confirms these findings. This effect mainly occurs when the father also smokes. Bynner (1969) did not distinguish between brothers and sisters, but in this study sisters have been shown to have little effect on boys' smoking compared with their brothers. On the other hand, for the girls the smoking of their sisters rather than of their brothers was the important factor. The sibling of the same sex would appear to have the greater influence on the children in our study.

The young smoker was more likely to say that his friends smoked, and this agrees with previous findings (Bynner, 1969; Bewley et al., 1974).

The academic achievement of smokers was lower than that of non-smokers, both as seen by themselves and by their headteachers. Fewer children thought their work was poor in contrast to the headteachers, who assessed one-third as poor. Girls generally ranked themselves lower than boys. These children are younger than many other groups that have been studied, but some of our findings support studies of older children (Hargreaves, 1967; National Health and Medical Research Council, 1969; Newman et al., 1970; Laoye et al., 1972) where children graded higher in academic ability smoked less.

The main reason for smoking in the children's view was to show off. None of the other reasons suggested was agreed by more than half the children, and curiosity was rarely mentioned. The children did not seem to enjoy smoking. Few of them agreed that smoking was enjoyable, and few thought children smoked because they like it. They did not think smoking was a desirable activity for children, and they also thought parents should discourage their children from taking it up. Most of the children in each group thought smoking was harmful to health, and they also thought that health worries were a deterrent. However, these views were held by fewer smokers than non-smokers.

The negative attitude of both boys and girls to smoking was also found in our previous study of primary school boys (Bewley et al., 1974). This does not conform with the findings of Bynner (1969) who 
reported that most of the smokers in his sample enjoyed smoking. This could be an age effect as the boys in Bynner's sample were older. The findings of Bland et al. (1975) suggest that boys between 10 and $12 \frac{1}{2}$ years do not identify themselves with smokers, despite their own smoking habits, and this may account for some of the discrepancies between attitudes and behaviour.

There was no evidence of a relationship between smoking and social class. Bynner (1969) found the same. McKennell and Thomas (1967) found that regular smoking for adolescents began earlier among social classes IV and V, than among I and II. Most of the children in our study came from social class III, so it would be difficult to detect any but large differences in the social class distribution with our smoking groups.

The study shows that starting to smoke at an early age is mainly a result of social factors. Children experiment with cigarettes and begin regular smoking despite being aware of the health hazards. Clearly, health education will have to take these social factors into account, but the problem is complicated as reasons for beginning to smoke vary for different groups of children. Anti-smoking education ought to be directed at children who are more at risk. If this is to be done, we need as clear a picture as possible of how and why children take up smoking. We hope that these findings add to this, and that with further work a better understanding of children's smoking will help health educators to discourage children from taking up the habit.

We should like to thank the children, their parents, and the staffs of the schools in the study for their help and co-operation. We also thank the staffs of the Kent County Health and Education Departments, particularly Dr A. Elliott (formerly County Medical Officer) and Mr P. O. Page, and members of the Department of Community Medicine, St Thomas's Hospital Medical School, particularly Miss I. Day, Mr T. Halil for the statistical design of the study, Mr R. Harris, Miss A. Clarke and Miss V. Pollard for statistical assistance, and Miss J. Price and Miss J. Chadwick for typing.

This study was supported in part by a grant from the Department of Health and Social Security.

Reprints from B. R. Bewley, Department of Community Medicine, St Thomas's Hospital Medical School, London SE1 7EH.

\section{References}

Bewley, B. R., and Bland, J. M. (1976). Smoking and respiratory symptoms in two groups of schoolchildren. Preventive Medicine, 5, 63-69.

Bewley, B. R., Bland, J. M., and Harris, R. (1974). Factors associated with the starting of cigarette smoking by primary schoolchildren. British Journal of Preventive and Social Medicine, 28, 37-44.

Bland, J. M., Bewley, B. R., and Day, I. (1975). Primary schoolboys: image of self and smoker. British Journal of Preventive and Social Medicine, 29, 262-266.

Bothwell, P. W. (1959). The epidemiology of cigarette smoking in rural school children. Medical Officer, 102, 125-132.

Bynner, J. M. (1969). The Young Smoker. Government Social Survey. HMSO: London.

Cartwright, A., and Thomson, H. G. (1960). Young smokers. An attitude study among schoolchildren, touching also on parental influence. British Journal of Preventive and Social Medicine, 14, 28-34.

Hargreaves, D. H. (1967). Social relations in a secondary school. Routledge and Kegan Paul: London.

Kimball, A. W. (1954). Short cut formulae for the exact partition of $\chi^{2}$ in contingency tables. Biometrics, 10, 452-458.

Laoye, J. A., Creswell, W. H., and Stone, D. B. (1972). A cohort study of 1205 secondary school smokers Journal of School Health, 42, 47-52.

Lemin, B. (1966). A study of the smoking habits of 18 year old pupils in six schools in Aberdeen. Medicat Officer, 116, 82-85.

McKennell, A. C., and Thomas, R. K. (1967). Adults' and Adolescents' Smoking Habits and Attitudes. Government Social Survey. HMSO: London.

National Health and Medical Research Council (1969). Report of a Survey into the Smoking Habits and Attitudes of Australian Schoolchildren. NHMRC: Australia.

Newman, I. M., Ang, J., Irwin, R. P., and Smith, J. M. (1970). Adolescent cigarette smoking. New Zealand Medical Journal, 71, 161-166.

O'Rourke, A., and Wilson-Davies, K. (1970). Smoking and schoolchildren. Journal of the Royal College of General Practitioners, 20, 354-360.

Palmer, A. B. (1970). Some variables contributing to the onset of cigarette smoking among junior high school students. Social Science and Medicine, 4, 359-366.

Public Health Department Study Group (headed by S. P. W. Chave and R. S. F. Schilling) (1959). The smoking habits of school children. British Journal of Preventive and Social Medicine, 13, 1-4.

Royal College of Physicians (1971). Report on Smoking and Health Now. Pitman: London.

Salber, E. J., Freeman H. E., and Abelin, T. (1968). Needed research on smoking: lessons from the Newton study. In Smoking and Health Behaviour. Edited by E. F. Borgatta and R. R. Evans. Aldine: Chicago.

Todd, G. F. (1972). Statistics of Smoking in the United Kingdom, research paper I, 6th edition. Tobacco Research Council: London. . ,

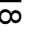

